\title{
Localization Techniques for Water Pipeline Leakages: A Review
}

\author{
Airull Azizi Awang Lah*, Rudzidatul Akmam Dziyauddin, Nelidya Md Yusoff
}

Razak Faculty of Informatics and Technology, Universiti Teknologi Malaysia, Jalan Sultan Yahya Petra, Kuala Lumpur, 54100, MALAYSIA.

\author{
*Corresponding Author
}

DOI: https://doi.org/10.30880/ijie.2018.10.07.028

Received 25 October 2018; Accepted 25 November 2018; Available online 30 November 2018

\begin{abstract}
Pipeline leakages in water distribution network (WDN) is one of the prominent issues that has gain an interest among researchers in the past few years. Time and accuracy play an important role in leak localization as it has huge impact to the human population and economic point of view. The complexity of WDN has prompt numerous techniques and methods been introduced focusing on the accuracy and efficacy. In general, localization techniques can be divided into two broad categories; external and internal systems. This paper reviews some of the techniques that has been explored and proposed including the limitations of each techniques.
\end{abstract}

Keywords: leakage localization, water pipeline, review, vibration, classifiers, residuals, sensors

\section{Introduction}

An efficient water distribution system relies on a good water pipeline system. Water pipeline leakages are one of a few challenges to the water utility companies all over the world in providing the service. Several factors have been identified contributed to the water pipeline leakages such as ageing infrastructure, severe environmental conditions and third-party damages [1]. A recent report published by World Bank in 2016 indicates there is 45 billion cubic meters of water loss in that year. From a financial point of view this huge amount of losses is equivalent to a value of USD 3 billion whilst from a social point of view it can supply water to 90 million people in a year [2]. During pipeline leakage, a portion of the water delivered within the water distribution network wasted and this affects the Non-Revenue Water (NRW) efficiency to the water utility company. NRW is the lowest hanging fruits to improve water utility efficiency [3]. Minimizing the NRW is one of the challenges encountered by the water utility company because the uncontrolled NRW can cause vicious cycle [4]. Researchers in [5] studied the detail of pipeline leakages and emphasized that there are two main factors that affecting to the NRW efficiency; apparent losses and real losses. The apparent losses were due to meter defect, illegal tapping, water storage tank maintenance, fire brigade use and others. The real losses were due to a pipe burst, pipe leakages and water overflow due to faulty valves. A statistic from [4] in 2008 exhibits that real losses accounted for $61.5 \%$ of total water loss recorded. Hence there is an urgency to detect the pipeline leakage and localize it in the shortest time with leak location accuracy is the main benchmark.

Pipeline leakage incidents sometimes go unnoticed until the symptoms are visible such as low water pressure or water supply interruptions. This happens when the water pipeline is located underground or far away from the community area. Even though the leakages is visible and noticed, it still takes time to the relevant authorities to locate the leak location. This issue has gained an interest in a lot of researchers and in the past few decades, numerous studies and research have been conducted to solve or at least minimize this issue. The research area in the field of localization can be partitioned into two categories; the external and internal system. The external system refers to the hardware-based such 
as sensors (acoustic, pressure and vibration), mobile wireless sensor, fibre optics, infra-red, ground penetrating radar and electro kinetic. The internal system refers to a software-based such as the use of classifiers, customized algorithm, event detection and methods to localize the leakage. Most of the internal systems use a pressure sensor to capture the data from water pipeline to be processed in the next stage.

The objective of this paper is to review previous localization methods and techniques proposed in recent years. Each method is discussed in terms of deployment and execution and the advantages and limitations. This paper also proposed a localization technique using vibration sensors. Section 2 presents the discussion review of the localization techniques and highlight the advantages and limitations. Section 3 proposed localization method and followed with a conclusion in Section 4 .

\section{Review Localization Methods}

The leakage localization methods can be broadly categorized into two, which are extenal system and internal system, as illustrated in Fig. 1.

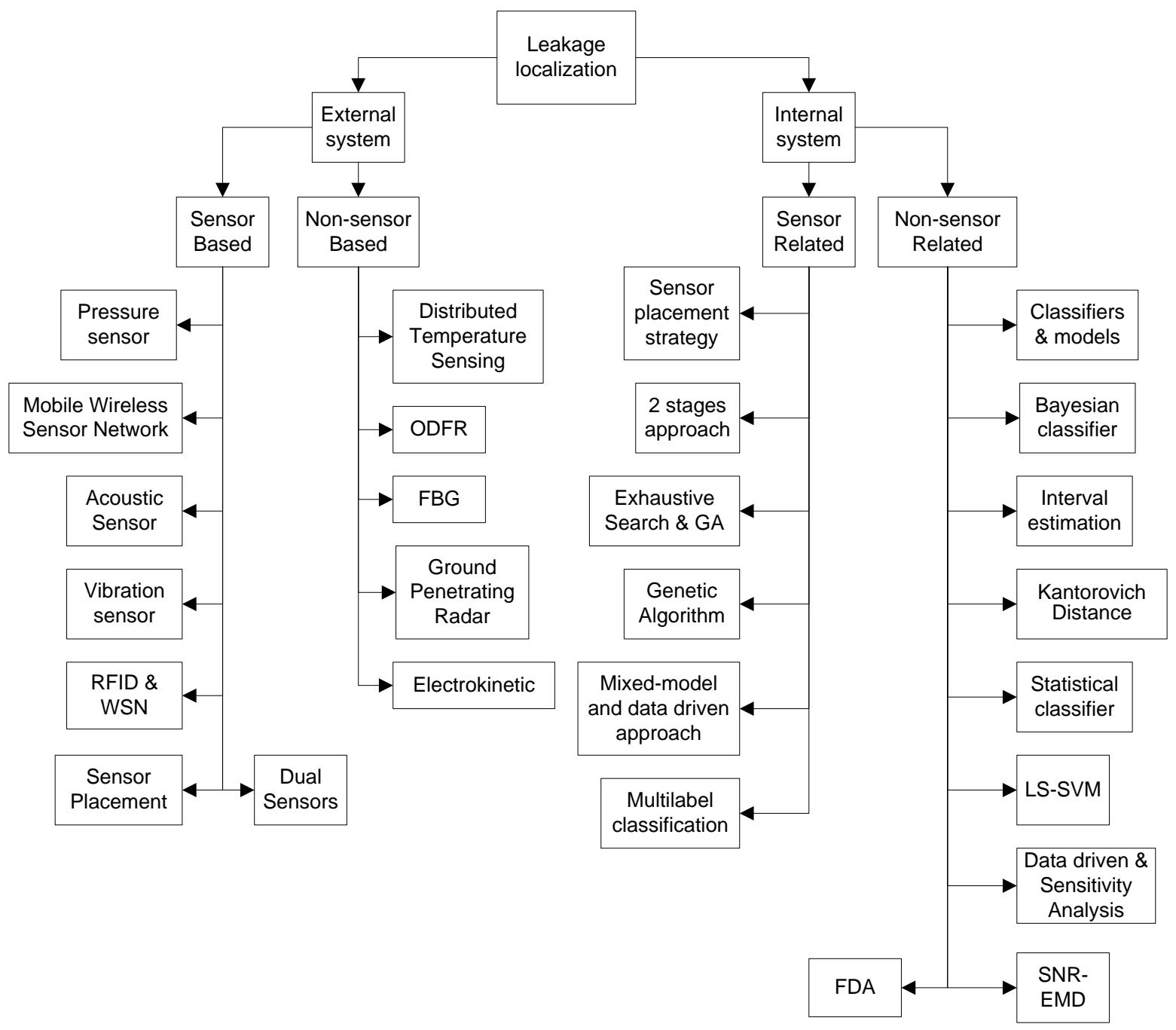

Fig. 1 - Overview of leakage localization techniques.

From a statistical point of view, leak detection is a detection problem while leak localization is an estimation problem. There are two categories of leakage localization techniques; the external system and the internal system. The external system is the use of external sensor attached to the pipeline captured the data and generated an alarm while the internal system is based on the application of numerous methods and techniques such as classifiers, mass-balancing, multi-label and residuals to process and analyze the data captured by external hardware. The internal system, in general, does not limit to the specific type of sensors [6]. 


\subsection{External Systems}

\subsubsection{Sensor Based}

Researchers in [7] combined the pressure and vibration sensors where the former functions to detect the leakage and the later functions to localize the leakage segment. The researchers utilize Head Loss Ratio (HLR) algorithm to detect the leak. The pressure readings to describe the leak detection method from three sensors labelled as $p_{i}, p_{j}$ and $p_{k}$ are shown in Equation 1.

$$
H\left(p_{i}, p_{j}, p_{k}, t\right)=\frac{p_{i}(t)-p_{j}(t)}{p_{j}(t)-p_{k}(t)}
$$

The first pressure signal is the inlet network pressure and the last pressure signal is the outlet of the network. The pressure in any point along the pipeline represented the middle value. Any leakage within the network can be estimated using these pressure values with the proposed numerical method. Leak localization can be determined using the HLR algorithm. All possible HLRs between three consecutive sensing locations in the network will be computed before and after the leak. A section where the leak has occurred is located using a suitable performance metric. When the leak has been detected, two vibration sensors are used to localize the leak location using cross-correlation techniques. Different leak distance from the vibration sensors will result in different times for the leak signal to arrive at the sensors. The speed of the sound in the pipe and sensors distance is used to measure the leak location using time differences. The vibration at different measurement points needs to be calculated in order to determine the two sensors located near the leak. Equation 2 shows the time series data for total signal available at node location $n$ :

$$
x_{n}(t)=s_{n}(t)+n_{n}(t)
$$

$s_{n}(t)$ presents a leak signal time series data is the time taken for a leak noise model propagating through the pipeline in a particular distance. $n_{n}(t)$ is a signal noise and it presents the vibration flow that occurs in the background. The researchers explained in details on the usage of this equation to localize the leakage using cross-correlation techniques in MATLAB. The proposed method herein does not require details of water pipeline parameters, such as water flow rates and pipeline diameter. From the experiment conducted, the localization error gives a different result due to the effect of temperature changes. This temperature changes the Young modulus and sound speed.

Mobile sensor networks have gained a lot of interest among researchers due to its nature and advantages especially for the deployment in a large area. It has been a favourable option because of its capability to locate the leak precisely compared to the existing methods without affecting the water utility operational service [8]. These advantages have been used as a basis in [8] to propose a leakage localization using a mobile wireless sensor network solution. The zone of interest in this research is part of water distribution network where the utility provider presumed a leak is present or an area which will be having a periodic monitoring. Sensors are inserted from a water distribution system junction and it will move freely within the pipeline following the water flow. The leak will be detected by a mobile sensor and the captured information is transmitted to the wireless access points located outside along the pipeline or after it is physically captured. Mixed Integer Nonlinear Programming (MINLP) were used to calculate the optimal sensor and beacon placement to maximize the accuracy and minimize the budget. The researcher proposed a split scheme due to MINLP complexity by separating the beacon and sensor placement problem. The beacon and sensor problem were calculated using the exhaustive search in linear time. The coverage and detection rate can be increased by having more sensors. Another work from [9] also uses Wireless Sensor Networks (WSN) integrated with Leak detection Predictive Kalman Filter (LPKF). PKF is a predictor combined with Kalman Filter to lower down the communication cost in WSN. Predictive Kalman Filter (PKF) algorithm was chosen because of its ability in data compression and leak identification (LPKF).

The clustering techniques are used to reduce the power consumption in WSN. The data were collected by the sensor nodes every 5 minutes interval in hourly basis with a sampling rate of $1000 \mathrm{~S} / \mathrm{s}$. The noise data were filtered and anomalies were detected locally by LPKF algorithm. The wave pressure changes when a there is a leak in the water pipeline. Two sensors were chosen where a leak occurs between the sensors. The leak detection calculation uses the concept of leak wave propagation and Time Difference of Arrival (TDOA) given by Equation 3:

$$
x=\frac{L-C \Delta t}{2}
$$

$x$ represents the leak distance from the nearest sensor node, the distance between the sensors represented by $L$, the wave propagation inside the pipeline is denoted by $C$ and $t$ is the pressure signal time difference arriving from the nodes that calculates the cross-correlate the signals. The LPKF measures the pressure variations and if it exceeds the threshold, a 
flag will be updated from 0 to 1 to indicate a leak. The researcher made known new techniques that were derived from the principle of leak wave propagation, and the arrival method time difference to localize the leakage. Force Sensitive Resistor (FSR) were used during the experiment to measure the pressure, however, the accuracies were not good enough. So far, this method is suitable to be used on-demand to monitor the water distribution network on a periodic basis or in the event of uncertain leakage occurrences either from consumer complaints or indicator from static sensors.

The acoustic sensor also gained an interest to some researchers in exploiting its benefit in localizing leakages in the pipeline. Unfortunately, the effectiveness drops when using non-metallic pipes or pipes larger than $300 \mathrm{~mm}$. To overcome this limitation, in the early 1990's the United Kingdom's Water Research Council concluded that the acoustic sensor must be passed along the pipeline to detect the leakage accurately. Works by [10] introduce a Sahara system to locate the leakages in water pipeline from leak noise correlators. The Sahara system uses an acoustic detector unit plugged into the main entrance at any tap point with a diameter larger than $50 \mathrm{~mm}$. During the process, the pipeline pressure must be maintained between 3 to 200 psi. The cable is inserted into the pipeline using an insertion, and it covered with a retractable guide to safeguard the cable from damage as it moved throughout the water pipeline. The umbilical deployment and retrieval are controlled by the winch and cable drum. The winch works to push the umbilical into the pipeline opposing the water pressure and to pull back the umbilical after performing the survey. During operation, the water flow carries the system throughout the water pipeline. During travelling along the pipeline, the detector head listens for any peculiar leak noise produced by under-pressure water that escaped from the leak in the pipeline. A noise will be generated in the event of a leak inside the pressurized pipeline and the noise magnitude and frequency depends on various factors such as pipeline shape, leak size, pipeline material and pressure. The sensor head stopped when a leak is detected. The acoustic sensor that moves along the pipeline will detect the greatest leak noise which indicates a leakage point. However, this method is time-consuming and labour-intensive [11].

Researchers in [12] explored the work of using accelerometer to calculate the vibration on the Acrylonitrile Butadiene Styrene (ABS) pipeline surface to detect pipeline leakages. The experiment objective is to quantify the MPU6050 performance in detecting the water pipeline conditions and differentiate the leak size by varying the water pressure using time domain analysis. In the study, MPU6050 vibration sensor was used instead of the acoustic sensor because acoustic leak detection is only suitable for a metal pipeline. Their research motivation is to explore the effectiveness of using a vibration sensor since most of the existing research and study is using pressure sensor [13-21]. The water pipeline testbed is using ABS pipe approximately 10 meters length and a 1-inch diameter. A water pump supplies water to the pipeline testbed and a valve is installed at the end of the pipeline to control the water pressure. The experiment was conducted with four different water pressure; $0.6 \mathrm{kgf} / \mathrm{cm}^{2}, 0.8 \mathrm{kgf} / \mathrm{cm}^{2}, 1.0 \mathrm{kgf} / \mathrm{cm}^{2}$ and $1.2 \mathrm{kgf} / \mathrm{cm}^{2}$ and three scenarios; No Leak, $1 \mathrm{~mm}$ leak and $3 \mathrm{~mm}$ leak. During the experiment, the vibration sensors installed on the water pipeline collected data from three axes; $\mathrm{x}, \mathrm{y}$ and $\mathrm{z}$. The sensor is connected to a laptop for data processing and analysis using a wireless Zigbee network. Fig. 2 illustrates the wireless sensor system block diagram. The distance between the leak and sensors is 1.5 meter. The vibration data were collected in 3 sets and the time taken for each set is 2 minutes.

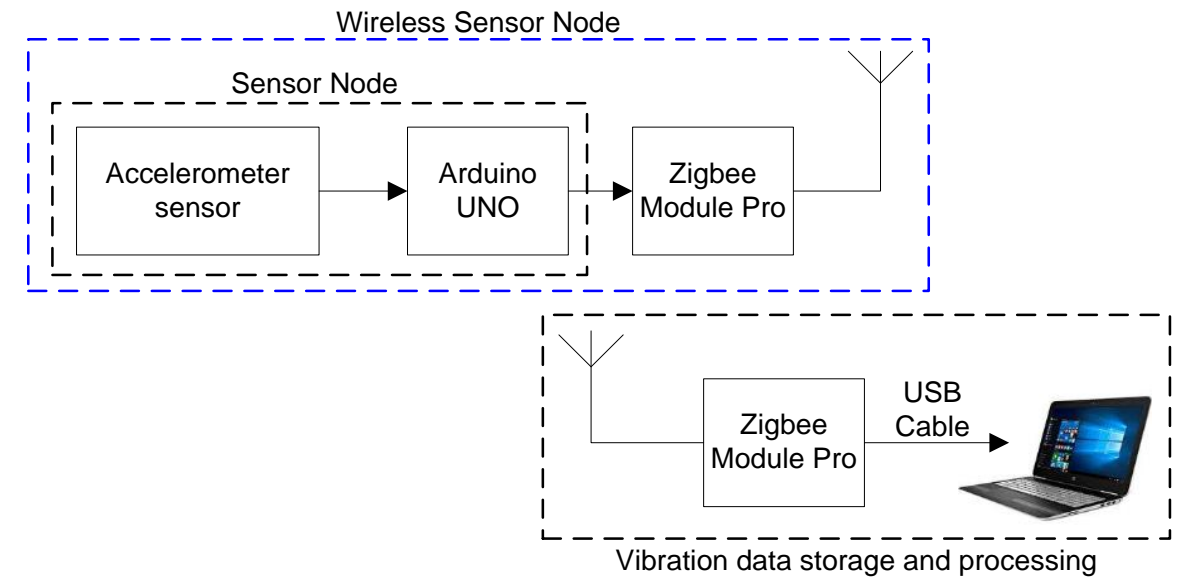

Fig. 2 - Wireless sensor system block diagram.

From the study, the researcher concluded that the MPU6050 can identify the water pipeline conditions across the three axes with varying water pressure. However, the water pipeline condition and leak sizes can only be recognized across $\mathrm{x}$ and $\mathrm{z}$-axis. In line with that, this paper will extend the work of using the accelerometer to localize the leakages by cross-correlating the signals from two vibration sensors. 
A combination of Radio Frequency Identification (RFID) and WSNs was proposed by [22] to monitor the water pipeline leakages in non-real time. The proposed solution was designed for a long-distance water pipeline that located above the ground. The researchers station a number of mobile wireless sensor nodes along the pipeline. The nodes are driven by the water flow from the pipeline source until the end where it will be collected and the data inside the memory is transferred to a computer for processing analysis. The RFID tags were installed outside the pipeline for localization purposes. The total RFID tags given as ' $M$ ' required is inversely proportional to the tag distance given as ' $\Delta d$ '. If the pipeline distance given as ' $D$ ', the total number of RFID tags required are:

$$
M=\frac{D}{\Delta d}
$$

Each of the mobile wireless sensor nodes operates following a preschedule instruction and to control the power consumption only one node is active while other nodes are in a sleep mode at a time. Three node wake-up techniques were used and the energy consumption and memory size requirements for the mobile wireless sensor node were calculated using a mathematical model that was derived for each of the technique. The wake-up techniques used are interrupt-driven, location-based and time-based. The mathematical models were analyzed and Matlab software is used to run the simulation. The interrupt-driven has lower energy consumption compared to the location-based and time-based. The location and time-based techniques also have limited scalability compared to the interrupt-driven. This was the effect of marginal activities that is running while the nodes are in sleep mode. The findings from this paper are more towards the development of energy efficient WSN for monitoring the water pipeline leakages. It does not focus on the leakage localization.

Leak localization is an optimization problem and there are numerous papers published about using sensors placed in a pipeline to formulate the leak localization. Nevertheless, very few focused on the sensor placement and this encourages [23] to propose a leak localization technique based on sensor placement strategy and demand uncertainties. The ambiguity in the hydraulic model has been overlooked, for example, variation in the demand causes stochastic deviations on the pressure in the system. This source of variations is referred to as process noise. From the process noise calculation, the sensitive point in leakage perspective is related to the sensitive part due to demand fluctuations. Therefore, the point is not sturdy and pragmatic. This emphasizes that for a robust sensor position, there is a need for uncertainty analysis in the hydraulic distribution system. Sensor placement can be represented in an optimization problem. The most common techniques have mounted the sensors at the sensitive location along the pipeline. The researchers focused on the fusion of process noise in the algorithm of the pressure sensor placement to localize the leakage in the water distribution network. Two techniques to calculate the process noise were proposed; Monte-Carlo Simulation (MCS) and Hydraulic Small Signal Model (HSSM). The pressure measurement standard deviations from demand fluctuations were calculated with MCS and then it is incorporated with non-binarized leak sensitivity matrix. The proposed techniques were tested in a network model from literature and real water distribution network located in Linz, Austria.

Distinguishing the interference signals from valve movements, pump regulating and pressure regulating can reduce the false alarms during pipeline leakage detections. Researchers in [24] proposed digital recognition algorithm for interference and leak signals using dual-sensor systems. The signal direction can be identified using a cross-correlation calculation between the two signals from the two sensors. The proposed algorithm overcomes the installation distance limitation without the need for high sampling frequency as compared to the dual-sensor method that was based on the delay of the hardware circuit.

\subsubsection{Non-sensor Based}

Distributed Temperature Sensing (DTS) technology is one of the commonly used methods to detect pipeline leakage. Preliminary works in DTS to localize the leakages in sewage pressure pipes were undertaken by [13]. The researchers utilize the DTS technology to measure the temperature along the fibre optic (FO) cable. A linear sensor represented by a laser pulse that transmitted from one end and propagates along the cables to the other end. The backscattered photon is measured using a spectrometer, and the backscattering location can be calculated by measuring the backscattered photon travelling time. The inelastic scattering shifts the wavelength spectrum into two components; Stokes and anti-Stokes. Stokes component shifts the wavelength into lower frequencies, and it is temperature independent while the anti-Stoke components shift the wavelength into higher frequencies, and it increases exponentially with temperature. In this study, the researchers used the Raman scattering to measure the temperature. The temperature can be deduced from the ratio of the two components magnitude. The number of photons used to calculate the ratio determines the temperature accuracy and therefore it is dependent on the measurement temporal and spatial resolution.

The experiment was conducted using Silica XT-DTS device with a maximum spatial resolution of 0.25 meter and a temporal solution of 10 seconds and a Brusens temperature Fiber Optic (FO) cable. The FO cable was installed permanently within the pipe protective sheathing. During the experiment, special focus was given in varying the cable 
positioning within the pipeline. The pressure pipe was made from U-shaped Polyvinyl Chloride (PVC) 6-meter length and a diameter of $20 \mathrm{~cm}$. The DTS cable was threaded into the pipe using taut wire. There is a calibration section with ice and a warm bath at both ends. The cable positioning was located in every $25 \mathrm{~cm}$ and three reference sections were used to calculate the calibration parameters. A local temperature drop transpired when there is a water intrusion from leakages into the pipe. Thirteen sets of measurement sequences were supervised under numerous measurement conditions which include a number of leakages and FO positioning. The thirteen sets of measurement sequences differed in the leakages size, leakages number, cable positioning, spatial and temporal resolution. Tests were conducted with multiple leakages of different sizes and different spatial resolutions. From the experiment, small leakages size of $4 \mathrm{~mm}$ can be detected based on the plotted graph. The graph amplitude varies as it is dependent on the number of leakages, temperature differences and integration time. The cable positioning also has an impact on the recorded temperature. The temperature varies in each location throughout the cable. However, these techniques encountered challenges when testing in the real world because of temperature gradients within the pipe system over a longer distance and cable placement. Despite DTS is well-proven technology that has the efficacy to detect very small leaks in a short time, it becomes complicated to measure minimum detectable leak size or guarantee a maximum detection time [25].

The Optical Frequency Domain Reflectometry (ODFR) is applicable to be deployed for pipeline monitoring because of high precision in distributed strain measurement. This prompt [26] to propose a new application to monitor leakage and corrosion based on OFDR technique. The corrosion level and location are shown by a hoop strain nephogram from the sensor array. The pipeline testbed consists of PVC pipeline with a total length of $57.2 \mathrm{~m}$ with seven leakages valves separated $8.2 \mathrm{~m}$ distance. There are seven test segments made of polyimide-coated optical fibre bonded in a circle to measure the hoop strain variations during the leakage. The fibre optics which is in unbonded segments and close to the test segments is set as a reference to compensate with temperature change. The pipeline model is pressurized to $20 \mathrm{kPa}$ before simulating the leakage process. The leakage event in the pipeline is produced by opening the valve rapidly. The inner pressure dropped from $20 \mathrm{kPa}$ to $0 \mathrm{kPa}$ during the leakage test. The strain variation at a frequency of $100 \mathrm{~Hz}$ is measured using ODiSI system throughout the testing period. The Distributed Optical Fiber Sensor (ODFS) able to detect the leaks during the leakage test, however, the leakage localization accuracy is low because of the limited sampling frequency of the ODiSI system.

Fiber Optic (FO) cable is one of the preferable choices for long distance because of its vulnerability to electromagnetic interference, high accuracies and multiplexibility. This advantages has inspired [27] to proposed leakage localization techniques by integrating Fiber Bragg Grating (FBG) and Background Propagation (BP) neural network classification algorithm. Method of Characteristics (MOC) is used to solve the leakage occurrences hydraulic transient process. The MOC method is used to calculate the hoop strain deviation in any nodes along the water pipeline. During the experiment, three leakages locations were simulated, the leakage distance is $5.5 \mathrm{~km}, 27.5 \mathrm{~km}$ and $49.5 \mathrm{~km}$ from the inlet. In general, the researchers concluded that a relationship between hoop strain variations and leak location can be established because different leak locations produced different hoop strain variations. The FBG hoop strains sensors is used as the sensing element is installed along the water pipeline to calculate the hoop strain deviation. A leak point induced a Negative Pressure Wave (NPW) that spreads along the pipeline. A pressure drop is detected when the NPW arrived at the sensor and with combination of two sensors, the leakage point can be calculated by identifying the NPW arrival time. The researchers then apply the BP neural network using multiple hoop strain characteristics to localize the leakage. There are various multilayer networks to train the weights of nonlinear differential function. BP neural network was chosen because of its advantages in terms of fault-tolerance, self-organized learning and self-adaptivity when processing huge data amount in long-distance pipeline monitoring. The pattern recognition to estimate the leakage points were obtained by using the input neurons from five hoop strain variations. From the result, it shows that different leak locations generate different hoop strain variations and this was further analyzed using a machine learning method to identify the leak localization. In this study, the researchers estimate the leakage locations using a series of hoop strains values composes eigenvectors. This study only focused on an ideal pipeline without considering external factors. There are various possibilities that need to be explored such as buried pipelines, overhead pipelines or subsea pipelines to enhance the neural network using hoop strain variations.

Ground Penetrating Radar (GPR) is one of the widely used inspection techniques in geophysical imaging for underground monitoring and exploration although this technique is commonly known as a time-consuming and an uncertainty to provide reliable leakage location results. On the other hand, Infra-Red (IR) technology has been used extensively to detect pipeline leakage apart from the constraints of the measured radiation is likely influenced by the object temperature, surrounding weather, soil and pavement surface conditions [28]. Researchers in [28] proposed a leakage detection and localization method by integrating the GPR and IR. The equipment used in data collections are MALA GPR and VarioCAM 400 IR camera. The researchers introduced two algorithms; the approximate and exact mechanism to predict the leak location in a water pipeline. The water pipeline leakages were detected using developed methods based on the image segmentation algorithm from the IR and GPR images. The methods consist of two tiers; data collection and image processing. The actual and simulated leaks in the buried pipe were identified using GPR technology and IR images. The GPR emits an electromagnetic wave which will be reflected when it encountered underground objects. The antenna received the reflected signals and produced a subsurface underneath profile. To expedite the processing in MATLAB, the IR images in IRBIS format were converted to JPEG format to expedite the processing time 
in Matlab. Each JPEG image is analyzed using developed seeded a region-growing algorithm to; 1) classify the image into leakage and non-leakage areas, 2) calculate the leakage areas and 3) measured the centroid of leakages using the Green's theorem method. After the leakage area and location have been identified, the pipe location is compared with the leakage location by overlaying the IR images with GPR images. This enables to diagnose the leaks whether it is from bottom, top or side of the pipelines. The estimated leakage areas margin errors are from $2.9 \%$ to $5.6 \%$. This study was exploratory and interpretative in nature; however, it was tested only on pipelines made from PVC and therefore it can be extended further for other pipeline materials.

Electrokinetic phenomena is a cross-coupling phenomenon that associates with relative movement between pore water together with electrical dispersed layer and the solid phase of the porous material. The hydromechanical events can be localized from the electromechanical source using the electrographic method. This inspired [29] to proof that electrokinetic nature from the electrical field can be linked with the fluid leakages in a well. The experiment setup used a voltmeter produced from BioSemi Inc. that was purposely designed for electroencephalography to measure the electrical response. BioSemi ActiveTwo data acquisition system was used to measure the electrode potentials. Two experiments were conducted; the first experiment is localized two data snapshots in the distribution of electrical potential that linked with seal rupture in a well and the second experiment is to demonstrate that a multichannel voltmeter linked to the electrodes on the ground surface can detect the pulse of water injection in a heterogeneous natural ground. A distinct advantage of this technique is it is hard to detect self-potential signals which is relatively small from the ground surface notably with the existence of electromagnetic noise.

\subsection{Internal Systems}

\subsubsection{Sensor Related}

A common problem when confining leaks in the huge water distribution network is leak mislabeling and a large number of possible leakage locations. Hence, [15] proposed a sensor placement strategy by applying optimization problem to set up the sensor placement and reducing the undesired effects with proper assumptions and taken into consideration the real-world application such as the adequate distance of the leak location. The researchers divided the leak location problem into two stages; leak location stage and sensor placement stage. The leak location methodology targeted to locate leaks within the District Meter Access (DMA) using pressure measurement collected from the network. A methodology was introduced to locate the leaks within a District Metering Access (DMA) by using pressure measurement gathered from network and the estimation by network hydraulic model. A numerical simulation in Epanet hydraulic solver was used to obtain the sensitivity matrix known as leak signature. The leak signature was matched with the residual vector of each node to determine which node has the highest probability to represent a leak. The relaxed isolation index is used in the sensor placement stage methodology to manage the real-world effects that influencing the water pipeline system, such as non-linearity, model uncertainty and sensor placement resolution. The methodology presented here is using a pressure sensor, but it can be replaced with other types of sensor with minor modifications. This can be extended into multiple leak scenarios and further methods have to be developed and the existing sensitivity matrix can be expanded further by considering the selection of these scenarios.

Another method to localize the leakage is using a multilabel classification approach as exploring by [17]. In this study, the researchers proposed a combination of the wireless sensor networks with pressure data to distinguish and locate the leaks on various locations in the water pipelines. The data performance was compared using label classification; RAkEL (random k-label sets), BRkNN (binary relevance k nearest neighbors) and BRSVM to detect and localize the leakage. The experimental setup was made from plastic water pipeline with a length of 80 meters and a radius of $1.5 \mathrm{~cm}$. Three units of the wireless pressure sensor, Electrochem PS1 were mounted on the pipeline at three different locations. The pump motor of 1.5 HP is used to generate water pressure. The pressure data were recorded in 6 seconds. The pressure data is used as an input and the leak occurrences are the output. Further analysis, a multi-label learning problem arisess because the inputs cannot be mapped to only one label or class. Two types of information, which are the pipeline conditions and the leaking point, can be retrieved from the pressure data captured by the sensors. From the experiment, $\mathrm{RA} k \mathrm{EL}$ is the best, however, the accuracy will drop if the leakage location located far from the pressure sensor because of the distance.

Researchers in [21] proposed leak localization method based on a mixed model-based and data-driven approach. The Pressure residuals were generated by monitoring the water network models and the models are implemented using Epanet hydraulic simulator. In the first stage, the pressure measurement was compared with the estimation provided by Water Distribution Network (WDN) to obtain the residuals. In a geometric framework, the raw residuals do not provide a simply fault confinement because all the residuals are affected by the leaks and there is a high level of ambiguity that the nodal demands and leak size is unknown because the noise affected the measurement taken. Thus, the raw residuals are proposed to feed the classifiers because in this situation the use of the classifier does not encounter problems associated with purely data-driven methods. The proposed method for offline design is based on four stages; modelling, data generation, grouping and classifier training and evaluation. The classifier can be used online to localize the leaks after it has been validated. In the second stage, the leak location is determined by applying the classifiers to the computed residual 
values. The WDN simulation under different uncertainty conditions and leak scenarios produced the data will be used to train the classifier. However, in the presence of uncertainty, this strategy may provide limited results. Therefore k-Nearest Neighbors $(k-\mathrm{NN})$ algorithm and temporal reasoning are applied to overcome the limitations. This method was developed for a single leak. For multiple leaks, the classifier needs to be trained to different possible multiple leak combinations, and it is time-consuming. Hence, there is a need to introduce another efficient method to address the multiple leak problems

Different approaches were explored by [19]. In here the researchers proposed a leakage localization in DMA of water distribution networks based on integrated model-based monitoring framework. The methodology is based on consolidation of flow and pressure sensor at the DMA inlets. Genetic Algorithm (GA) optimization is used to calculate the sensor placement. The problem of sensor placement was solved using the optimization-simulation framework and thus enhancing the leakage localization overall performance. Fig. 3 illustrates the proposed framework.

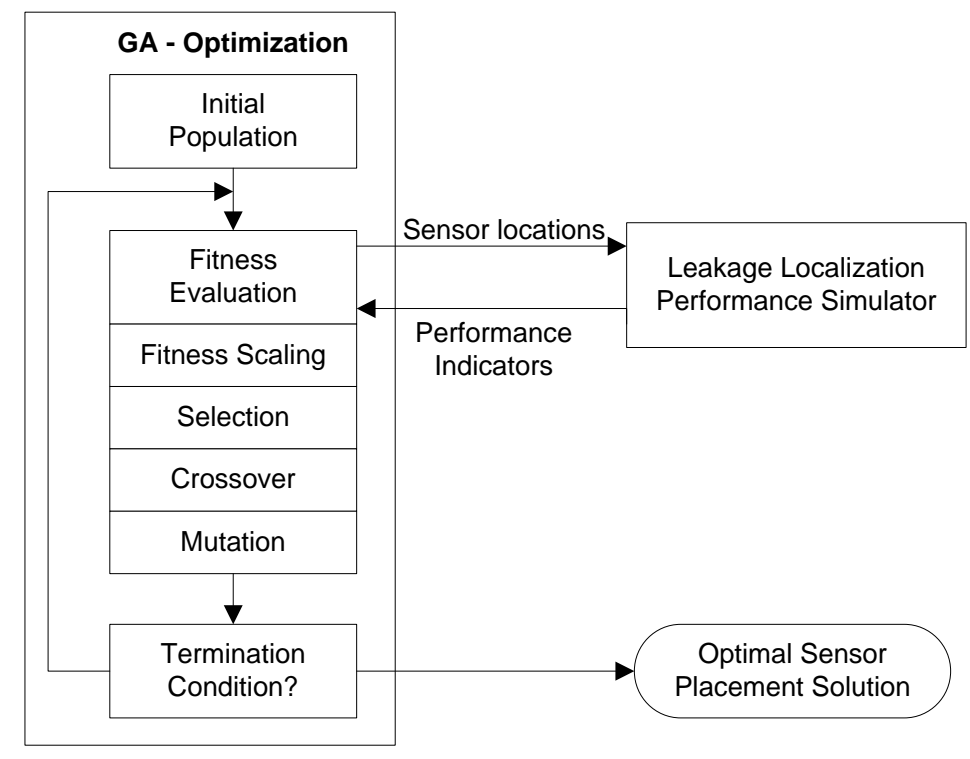

Fig. 3 - Optimization simulation framework.

This methodology is based on the process of GA-based optimization-simulation and leverages the benefits of GA's comprehensive search capacity to develop a potential solution (sensor location) population to the near optimal value. The 'Fitness Evaluation' step simulation measured the proposed potential solution (sensor locations) fitness. The DMA pressure sensor location plays an important role in determining the leakage localization performance in the context of leakage detectability and isolability. The pressure sensor placement method based on binary were also considered in order to detect either there is a leak or not. However, this does not reflect the real network where the pressure sensitivity is dependent on its location. For example, a pressure sensor that is near to the DMA inlets will record a low-pressure sensitivity because the pressure was set by the pressure reducing valves. A direct modelling approach was integrated to identify the leak locations. This study focused on the occurrence of single faults inside the water distribution network. It can be expanded to consider a case of multiple concurrent faults.

A different technique was proposed by [30] to detect the fault and localize small leakages in water distribution systems. The techniques proposed identified the positions of flow meters and reducing valves installation nodes. The identified positions are used further to acquire the information needed. The researchers utilize two approaches; exhaustive search and genetic algorithm combined with economic and sensitivity measures to produce the objective and fitness function. The proposed techniques were tested in a real-world water distribution network and the result obtained is fairly accurate.

A calibration-based problem solved using a two-stage approach is introduced by [31] in the field of pipeline leakage localization. The values of the calibration parameters are identified by solving the optimization problem using Genetic Algorithm. The method proposed takes into consideration the nodal leakages, a pipeline with incorrect roughness and unknown valves status. This new method minimizes the solution search space size in the optimization problems. The scenarios of planned hydrant discharge during low demand period was simulated using hydraulic modelling to generate artificial calibration data. The method is tested in different leakage scenarios. Since optimization is one of the effective tools in detecting the leakage location, the combination with a quality field data and systematic approach that relies on hydraulic models is beneficial to the operations side. Estimating calibration data with sufficient confidence need a huge amount of accurate observation data, but this does not happen in a real-world scenario because in reality the collection 
of field measurement data is constrained by the time and financial limitations. The localization model accuracy is affected by the noise from the unknown small leaks and valve opening and closing..

Researchers in [18] proposed a new algorithm to detect the leakages in water distribution network using a pressure sensor. The proposed method solely involved pressure data without the water flow rate and it overcomes three problems that occurred in small leakage detection: inadequacy of durability under noisy conditions, shallow accuracy due to insufficient confidence bound and time information loss. The proposed method is a combination of floor function and shifted pressure data. The floor function consists of three parameters followed by a function of curvature and statistical estimation to localization. In the graph analysis, the burst pressure data shape is identical to the leakage pressure data, however, the leakage pressure change size is lower than the burst pressure change. The following process is recommended to attain the leakage case data.

$$
P_{\text {leak }}=K_{\text {factor }} \times P_{\text {burst.e }}+\left(1-K_{\text {factor }}\right) \times P_{\text {burst.e }}(0)+\text { noise }
$$

1) Use Kalman filter (KF) to isolate the recorded pressure data into pressure estimate $\left(P_{\text {burst.e }}\right)$ and noise.

2) The scaling factor, $K_{\text {factor }}$ is multiplied by $P_{\text {burst.e }}$ and add $\left(1-K_{\text {factor }}\right) P_{\text {burst.e }}(0)$.

3) Add the noise which was isolated in step (1).

This method reconstructs the burst data into artificial leak data. The filtered pressure $\left(P_{\text {leak }}\right)$ sampled every $T \mathrm{~s}$ is obtained using KF after producing the leakage case $\left(P_{\text {leak }}\right)$ pressure data. The leak case pressure is denoted by $P(k)$, model noise represented by $w(k)$, the measurement is represented by $z(k)$ and noise measurement at $k T \mathrm{~s}$ is denoted by $\mathrm{v}(k)$. The existing pressure can be considered as similar to the pressure in an earlier time because of very short interval of the sampling rate. Equation 5 summarized this.

$$
\begin{aligned}
& P(k+1)=P(k)+w(k) \\
& z(k)=P(k)+v(k) \\
& w \sim N(0, Q), v \sim N(0, R), P(0) \sim N\left(0, C_{0}\right)
\end{aligned}
$$

In Equation 6, the covariances of $w(k)$ and $v(k)$ is denoted by $Q$ and $R$ respectively and the initial estimate covariance is $C_{0}$. The proposed model considered accurate because the $Q$ value is set comparatively smaller than $R$ and most of the error was due to measurement error. The value of $R$ is determined after observing the measurement noise. Since the initial value was assigned from initial pressure measurement, the value of $C_{0}$ is similar with $R$. It is necessary to correct the average pressure value to zero because of different in pressure range measured in each sensor. Then, the mean value is deducted from the measured pressure.

$$
\begin{gathered}
P_{m}=\sum_{i=0}^{j} P_{\text {leak.e }}(i) \\
P_{\text {shift }}(k)=P_{\text {leak.e }}(k)-P_{m}
\end{gathered}
$$

Prior to an occurrence of leakage in a normal state, it is feasible to discover the pressure profiles near to zero by using the mean-shifted pressure value $\left(P_{\text {shift }}\right)$. This process alleviates the pressure data non-standardized variation due to consumptions. It is challenging to differentiate the change in water pressure due to leakage from erratic disruption, such as ordinary water consumptions. After a pressure drop when a leak occurs, the reduced pressure presumes to be fixed, therefore, this pattern can be represented by Equation 9.

$$
P_{C I}(k)=\sum_{i=0}^{k} P_{\text {shift }}(i) T
$$

The minor pressure drop caused by the disturbances can be neglected in the floor function with three parameters and it can be seen by the reducing $P_{C I}$ trend caused by the leak.

$$
P_{\text {floor }}(k)=\frac{P_{C I}(k)}{P_{m} / 10}
$$

From Equation 8, the denominator is $P_{m} / 10$. It means the result will be a significant change if the shift in the cumulative integral in Equation 7 is more than $10 \%$ of $P_{m}$. The user can set the portion based on the normal state observation. $\Phi$ is defined as the time minimal threshold value under the normal condition where the floor function value is fixed and the threshold number, $N$ is reduced. If the $P_{\text {floor }}$ continuously decreases in $C$ times in the interval of $\psi_{i}=(i=1,2, \ldots . C)$ and $C \geq N$ and $\psi \ll \phi$ for all $i$ then it indicated leakage is detected. These restraints are able to minimize the false alarm, 
but unable to resolve accurately the source of pressure change either it is due to water consumption or pipeline leakage. Under a normal state observation, users can control the two variables magnitude. The three parameters; $P_{m} / 10 \mathrm{~m}, \phi$ and $N$ are independent of each other. The denominator indicates the segment frequency where the floor function has similar value is determined by the burst or leakage size that a user want to distinguish.

\subsubsection{Non-sensor Related}

A localization technique that fuses together the use of classifiers and models based on data-driven approach with the use of statistical classifiers working in the residual space has been studied by [16]. The researchers localize the leak in water distribution network using pressure measurement and assessment from a hydraulic network model. The leak localization method was derived based from the comparison between monitored pressure disturbances due to leaking in certain nodes in the DMA and the DMA network model produced the theoretical pressure and disturbance from all potential leaks. The fault diagnostic is depicted in Fig. 4.

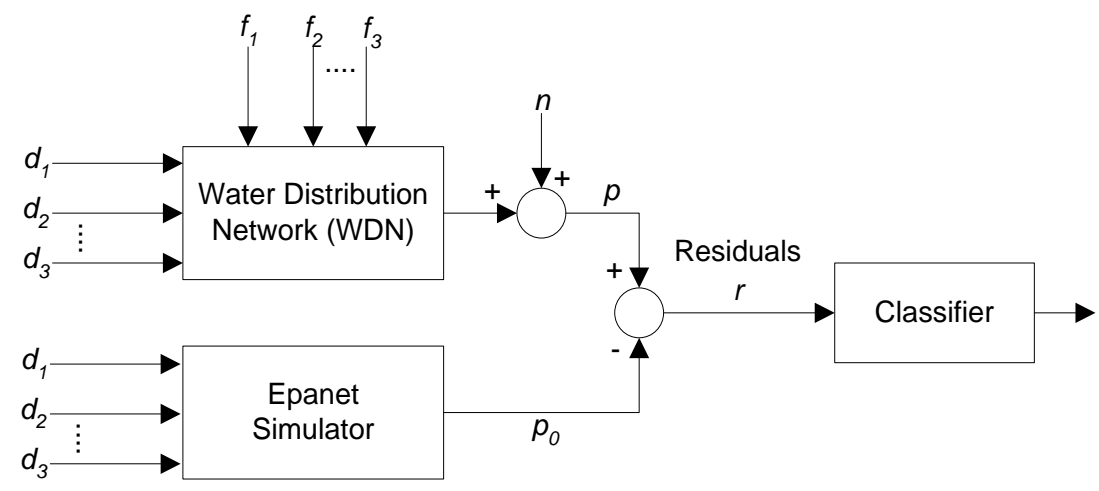

Fig. 4 - Fault diagnostic approach.

The residuals were created using the models and it was analyzed using the classifiers by considering the sensitivity of the residual leak. The hydraulic model was simulated using Epanet. The most apparent leak that generated the mismatches between the estimation and measurement determined by the classifiers. On identifying the leakage localization, there might be certain scenarios where the leaks appear in different locations. To overcome this, the proposed method with a classifier is repeated again.

Researchers in [21] proposed another localization method using Bayesian classifiers [20]. Although there is a work on leak localization using the Bayes theory, the researchers are exploring the use of Bayesian classifier in their works. The hydraulic simulator was used to calibrate offline the pressure residuals probability density functions for all possible leak scenarios considering the demand and leak size uncertainty sensor noise. The computed residuals were applied with Bayesian classifiers to determine the leak location in the WDN. Fig. 5 illustrates the leak localization scheme based on calculating the pressure residuals and use Bayesian classifier in the analysis.

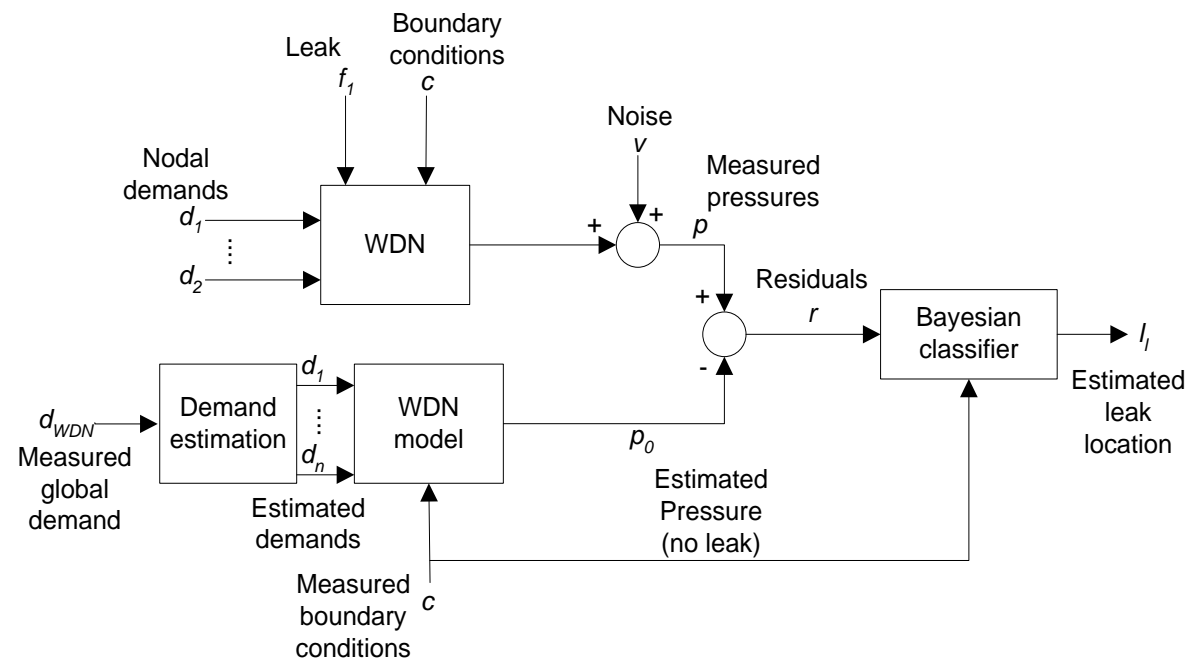

Fig. 5 - Leak localization scheme. 
Epanet hydraulic simulator is used to build a hydraulic model based on the DMA structures including the nodes, pipes and valves and the water pipeline network parameters such as pipe coefficients. The residuals $(r)$ are measured as a difference between data provided from the pressure sensors located along the DMA $(p)$ and estimation data from hydraulic model simulated under leak-free conditions $\left(p_{0}\right)$. The corresponding calibration process using real data, the model represents with accuracy the WDN behavior. The model is fed with estimated water demands since in practical nodal demands are not measured. Therefore, the residuals are not only responsive to faults but also to the disparity between the real demands and predicted values. Furthermore, the pressure measurement is affected by the sensor noise and this further affects the residuals. Considering all these factors, the Bayesian classifiers should have the ability to identify the real leak within the WDN and this leak can be located in any nodes and in any sizes and magnitudes. Besides, it must also able to withstand from noise and demand uncertainty. Some boundary conditions have limited the network operation. The simulation should take this into consideration and used as an input to the classifier. The researchers also improve the location accuracy by combining the Bayesian classifier and time-based reasoning. The findings focused on the leakage localization solely and the work can be extended for optimal sensor placement.

A considerable amount of literature [32] [33-34], when a leak is detected, the suspected whole ground area is dig up compared on focusing in one point. Considering this, the researchers proposed a localization technique that estimates the location of the leak as a partition with confidence bound. With wave speed is assumed to be constant, the leakages are presented in two variables; leakage location $\left(L_{e a k}\right)$ and time of occurrences $\left(t_{o c c}\right)$. This reduced the degree of freedom to 1 and either only $t_{o c c}$ or $L_{l e a k}$ is needed. Researchers in [35] calculate the wave speed in order to determine the leak location. Changes in the wave speed only resulting in a small error in the predicted location. The change of wave speed is negligible in the context of addressing the location of the leak in the segment. The proposed algorithm has distinct advantages in terms of high accuracy in leak localization and minimizing the false alarms.

Kantorovich Distance (KD) is minimum distance represented in terms of cost to shift mass of one probability distribution to another. A study by (Arifin) has proposed an algorithm based on KD to detect and localize leakage in pipeline. A pipeline exhibit 'leak-signature' when leak occurs. Leak signature has two characteristics. The first signature is pressure reduced in all nodes and the second signature is the mass flow rate increases in the first node and decreases in the last node compared to the nominal flow rate. According to the researchers this method capable to detect and localize leak size up to $1 \%$ from the nominal flow rate in the industrial pipeline. The proposed solution is much faster compared to the existing method in leak detection and localization. Although the proposed algorithm is unable to accurately locate the leak point, it is able to confine a small portion from the large pipeline that is leaking. This reduces the time and effort to the plan worker to rectify the leakage incident

A relationship between small leakages and pipeline inner pressure chaos characteristics were investigated by [36]. This relationship is used in the study of small leak detection method based on chaos characteristics and Least Squares Support Vector Machine (LS-SVM). The initial stage is to find the chaos in the pipeline inner pressure. Nonlinear time series analysis package (TISEAN) is used to estimate the relevant chaos characteristics. A Hybrid Kernel LS-SVM (HKLSVM). The purpose of HKL-SVM is to analyze the chaos characteristics and distinguished the Negative Pressure Waves (NPWs) were distinguished caused by small leaks. A simulation between HKLS-SVM and other models were executed using real pipeline data. The models are Gaussian kernel with LS-SVM (GLS-SVM), Sigmoid kernel with LS-SVM (SLS-SVM) and Polynomial kernel with LS-SVM (PLS-SVM). The pipeline data were obtained from the testbed setup that consists of pipeline length of 800 meters and the inside diameter is $200 \mathrm{~mm}$. There are two pressure sensors located at the pipeline entrance and exit. The pipeline was filled with seawater throughout the experiment and the water flow velocity is $1.5 \mathrm{~m} / \mathrm{s}$. The pipeline inner pressure is between $1-3 \mathrm{MPa}$. The small leak condition is simulated by opening and closing the pipeline valve. HKLS-SVM provide a good performance by leakage identification accuracy of $97.38 \%$ and leakage position accuracy of $99.28 \%$.

A leakage localization in water distribution using data-driven models and sensitivity analysis were proposed by [37]. In the paper, the researchers used reduced network models to isolate the lakes in water supply network. With reduced order network models, the network expected behavior can be predicted and then it can be compared with actual measurement recorded from the network. Based on the comparison results, the researchers produce a set of residuals which is used to isolate a leakage to a network node. The sensitivity matrix that captures the residual sensitivities to leakages is used in the leakage localization. The reduced order model is plug-and-play commissionable because it is adaptive based on measurement from the network. The sensitivity matrix calculation is performed offline using Epanet network model. The results show that the leakage can be isolated to a limited set of candidate vertices which contains the leaking vertex. In addition, the most likely candidate vertex appointed using the method is generally close to the actual leaking vertex. The proposed method optimally performed when the flow in the system is high. However, the proposed algorithm can be fine-tuned further because a few false alarms appears during the execution of non-leakage data. In addition, additional parameters can be included such as pipe ages because the older pipe has a high tendency to break compared with a new pipe.

A model-based leakage localization was proposed by [38]. In this study, the researchers investigate the impact of distinct parameter space sorting and different distance metrics on the objective function shape in model-based leakage localization. The gradient-based algorithm in leakage localization has a tendency to fail because the problem fitness landscape consists of many local optima. Therefore, an optimization problem with the heuristic algorithm is used to 
formulate the leakage localization. The disparity between the hydraulic simulation and real-world data were derived from the leakage simulation represents the objective function and this objective function is minimized to measure the leak location. The optimization problem is affected by the sorting method and ordering of the parameter space. The algorithm convergence speed evaluates the distance metrics and sorting and the results accuracy is measured based on the topological distance from the leak calculated using the algorithm to the actual leak. The proposed algorithm was assessed on a hydraulic model in the real water distribution network and it shows that search space Cuthill-McKee ordering performs in optimum level when used together with correlation distance metric.

Noise in the pipeline pressure has smaller amplitude compared to the pressure signal and it affects the pressure drop producing false alarm and further reduce the leakage localization accuracy. Researchers in [39] proposed a method named as Small Noise Reduction method based on Empirical Mode Decomposition (SNR-EMD) to minimize the noise in the pressure signal. The pressure signal means the line is measured by the signal's lower and upper envelope and the noise is the small fluctuations around the mean line. The Extreme Mirror Extension (EME) restrained the SNR-EMD end-effect. The SNR-EMD able to minimize the small noise by preserving the original signal mutation characteristics and making the denoised signal. The leak location in the pipeline is identified by combining the Negative Pressure Wave (NPW) with SNR-EMD. From the experiment conducted, the pressure drop can be recognized and leakage location is identified.

Fisher Discriminant Analysis (FDA) is used as a basis by [40] to solve the leakage localization problem in water distribution system. The proposed localization techniques consist of two parts; using the data from pressure measurement and applying the FDA to localize the leak. In the second part, the researchers focus on sensor placement problem when FDA is used to localize the leak. This technique was tested in Hanoi water distribution network (WDN). The WDN has 31 nodes in total and the sensitivity matrix was computed based on a leak of 50 litres per second magnitude. The simulation was run over a period of 24 hours and 15 minutes sampling time. In total there are 97 samples. With the use of two pressure sensors, the FDA method able to locate all the 31 leaks in the correct node. It also outperforms the angle method in the optimal sensor configurations.

\subsection{Leakage Localization Summary}

Table 1 summarizes the comparison of leakage localization techniques. From the list of localization techniques, vibration sensor has distinct advantages compared with others. This was clearly explained in [12] where the vibration sensor performs well in non-metallic pipes example ABS and PVC. Non-metallic pipes have viscoelastic properties that reduce the sound waves and absorb the sound energy. The air presence within the pipeline affects the leakage detection accuracy. The benefits of vibration sensor also have attracted researcher in [7] to use it in leakage localization. However, their study implements two types of sensor instead of one.

Table 1 - Comparison of leakage localization techniques.

\begin{tabular}{lllll}
\hline No & Techniques & Advantages & Limitations \\
\hline 1 & Distributed & Optical & Suitable for long-distance pipeline. & Low leakage localization accuracy. \\
& Fiber Sensing & $\begin{array}{l}\text { Hoop strain nephogram has good } \\
\text { performance in identifying the corrosion } \\
\end{array}$ & & \\
& & scope and evaluating corrosion degree. &
\end{tabular}

2 Fibre Optics \& BP Suitable for long distance water pipeline. neural network. $\quad$ No potential safety hazard.

High accuracy and multiplexibility, and Invulnerability to interference from electromagnetic radiation

Fault-tolerance, self-adaptivity and selforganized learning when processing huge data amount in monitoring the longdistance pipeline.

3 Ground Penetrating High accuracy with margin errors between Radar and Infra-Red $\quad 2.9-5.6 \%$ in estimating leakage areas

4 Pressure Sensors \& A pressure sensor to detect leakage and Vibration Sensor vibration sensor to localize the leaks.

5 Mobile Wireless No disruption to water utility service Sensor Networks during locating the leaks.

6 Wireless Sensor Network with LPKF
Cost economical for long distance water pipeline
The study focused on the ideal pipeline. Need to explore in details for various possibilities such as buried pipelines, overhead pipelines or subsea pipelines in order to evaluate and enhance the neural networks using hoop strain variations

Only tested only in PVC pipes.

Time-consuming and labour intensive.

Combinations of two methods to detect and localize the leaks.

FSR sensor was less accurate.

This method is suitable for the ondemand request.

Limited to water pipeline above the ground. 
7 Electrokinetic and Filled the research gap of using Genetic Algorithm

8 Acoustic Sensor

9 Vibration Sensor

10 RFID and WSN

11 Sensor placement based on demand uncertainties

12 Interference digital recognition from Dual sensors

13 Sensor Placement Strategy

14 Statistical Classifier

15 Bayesian Classifier

16 Pressure sensor and Genetic Algorithm

17 Multilabel Classification and wireless pressure sensor

18 Pressure sensor and Interval Estimation

19 Mixed-model and data-driven approach and pressure sensor

20 Kantorovich Distance

21 Chaos characteristics and Least Square Support Vector Machines (LS-SVM)

22 Data-driven models and sensitivity analysis

23 Differential evolution algorithm electroencephalography

Suitable to detect and localize leakages for pipes larger than $300 \mathrm{~mm}$ or on nonmetallic pipes

Able to identify the water pipeline condition and leak size across three axes for water pressure range from 0.6 to $1.2 \mathrm{kgf} / \mathrm{cm}^{2}$.

Suitable for a long-distance pipeline.

Low WSN power consumption.

Leak localization is fairly accurate

No need high sampling frequency

The same methodology can be applied to another sensor types with minor modifications

Overcome the limitation from the usage of models and classifiers by combining both methods to detect and localize the leaks.

Fill the research gap of using Bayesian classifier for leakage localization.

Simple deployment.

Use of wireless pressure sensor suitable for deployment in the large area.

Overcome three problems that occurred in small leakage detection: inadequacy of durability under noisy conditions, shallow accuracy due to loss of time information and lack of confidence bound.

Cheap, easier to install and maintain

Efficient in handling transient's process by faster detection and localization compared to existing statistical methods.

Designed to detect and locate small leaks.

Leaks can be isolated using the sensitivity matrix.

Cuthill-McKee ordering is better than Alphabetical and Random sorting as it is able to recognize $70 \%$ of the leak.
Challenges to detect self-potential signals especially in the presence of electromagnetic noise.

Time-consuming and labour intensive

The study in leak detection can be extended to leak localization by using two vibration sensor.

Limited to pipeline located above the ground.

Does not focused on leakage localization efficiency.

MCS method requires high computation time

Not cost effective as four unit of sensors is needed

Signal direction cannot be deduced if cross-correlation coefficient $=0$.

Focus on a single leak, the sensitivity matrix needs to be expand taking into account another leakage scenario.

Need a second round of analysis with classifiers if the leaks identified associated with more than one locations

Focused on the leakage localization solely and the work can be extended for optimal sensor placement.

Need to consider the case of multiple concurrent faults

Accuracy reduces if the leakage location located far from the pressure sensor.

Only focused on the accuracy of three labels classification, not on the leakage localization.

Leak location estimation accuracy depends on the wave speed.

For multiple leaks, the classifier needs to be trained to different possible multiple leak combinations and it is timeconsuming

The algorithm parameters can be tuned further to have more real-time results.

Slower computation cost compared to Back Propagation Neural Network (BPNN)

The method performs effectively when the flow is high.

Proposed algorithm needs to fine tune further because of false alarm during processing the non-leakage data.

Leakage localization accuracy depends on the distance metric and leakage position order. 
24 Small Noise Reduction based on Empirical Mode Decomposition (SNR-EMD)

25 Sensor placement based on exhaustive search and GA

26 Fisher Discriminant Analysis (FDA)

27 GA and two stages approach
Low cost and simple operation

Less complexity as the proposed techniques left aside the noise effect in the signal.

FDA method able to locate all the leaks with two pressure sensors.

Multiple optimizations prove beneficial to obtain reasonable representation for the number of leaks.
The proposed method can be fine-tuned further to improve the leakage location accuracy by calculating noise from other factors.

Focused on predefined areas where a leak might occur.

Apply appropriate performance indices for large-scale deployment.

Angle method outperforms the FDA method in three sensors configurations.

Works can be extended on using real field data

Accuracy depends on number and locations of available measurement in large WDN.

\section{Conclusion and Future Works}

Water leakages has a serious impact to mankind as the impact not just to the human population, but to the economic as well. Time and accuracy are an essence in detecting and localizing the leakage. Numerous papers on leakage localization techniques have been published in the past few years. Though it starts with a leak detection, nevertheless localization has gained an interest among the researchers as it brings more benefits compared to the leak detection. In overall, leakage localization techniques can be divided into two broad categories; the external and internal systems. To localize a leak, the effect is not just coming from the internal element, such as noise from valve and water pump, but from the outside element as well as such as water demand from the users. Each localization technique has its own advantages, limitation and accuracies depending on its surrounding deployment. WSN's based system is widely used in a large WDN. However positioning the WSN's must be taken into consideration as well which prompt a new branch of study that focused on the optimum positioning in WDN. Other than the positioning, eliminating the unwanted noise also plays a crucial role. Noise is one of major influence in the localization accuracy and this led to the research and investigation to minimize it. Some techniques have the accuracy and scalability, but the drawback is on the processing time which does not practical in real world implementation. Regardless of that, looking from other perspectives, all the contributions in the leak localization provides more understanding and expand the knowledge boundaries on the wave signal characteristics and properties in the pipeline and reduce the number of assumptions. This creates more possibilities for the development of efficient leakage localization techniques and methodologies.

\section{Acknowledgements}

This research was funded by Universiti Teknologi Malaysia research grant Q.K130000.2740.00K70 and Ministry of Higher Education under Fundamental Research Grant Scheme (Vot. No: 4F936).

\section{References}

[1] Adedeji, K.B., Y. Hamam, B.T. Abe, and A.A. Abu-Mahfouz, Towards Achieving a Reliable Leakage Detection and Localization Algorithm for Application in Water Piping Networks: An Overview. IEEE Access, 2017.

[2] Bell, C.M.W.P. The World Bank and the International Water Association to Establish a Partnership to Reduce Water Losses. 2016; Available from: http://www.worldbank.org/en/news/press-release/2016/09/01/the-world-bank-andthe-international-water-association-to-establish-a-partnership-to-reduce-water-losses.

[3] Hvilshoj, S. Reduction of Non-Revenue Water Around the World. 2014; Available from: http://www.iwanetwork.org/reduction-of-non-revenue-water-around-the-world/.

[4] Malcolm Farley, G.W., Zainuddin Bin Md. Ghazali, Arie Istandar, Sher Singh, The Manager's Non-Revenue Water Handbook. 2008. 110.

[5] Mijares, L. Water Audit Helps Identify Non-Revenue Water Losses. 2015; Available from: http://www.waterworld.com/articles/print/volume-26/issue-80/editorial-features/water-audit-helps.html.

[6] Geiger, G., D. Vogt, and R. Tetzner, State-of-the-art in leak detection and localization. Oil Gas European Magazine, 2006. 32(4): p. 193.

[7] Saqib, N.U., M.F. Mysorewala, and L. Cheded, A Multiscale Approach to Leak Detection and Localization in Water Pipeline Network. Water Resources Management, 2017. 31(12): p. 3829-3842.

[8] Gong, W., M.A. Suresh, L. Smith, A. Ostfeld, R. Stoleru, A. Rasekh, et al., Mobile sensor networks for optimal leak and backflow detection and localization in municipal water networks. Environmental Modelling \& Software, 2016. 80: p. 306-321. 
[9] Karray, F., A. Garcia-Ortiz, M.W. Jmal, A.M. Obeid, and M. Abid, EARNPIPE: A Testbed for Smart Water Pipeline Monitoring Using Wireless Sensor Network. Procedia Computer Science, 2016. 96(Supplement C): p. 285-294.

[10] Mergelas, B. and G. Henrich, Leak locating method for precommissioned transmission pipelines: North American case studies. Leakage 2005, 2005: p. 12-14.

[11] Gupta, S.P., A. Mahalwar, and P. Udaykumar. Analysis of different techniques for locating leaks in pipes in water distribution system using WSN. in Computational Intelligence on Power, Energy and Controls with their impact on Humanity (CIPECH), 2014 Innovative Applications of. 2014. IEEE.

[12] Ismail, M.I.M., R.A. Dziyauddin, N.A. Ahmad, and N. Ahmad, Vibration detection in water pipelines leakage using wireless three-axis accelerometer sensor. International Journal of Advanced Science and Technology, 2018. 112: p. 137-150.

[13] Apperl, B., A. Pressl, and K. Schulz, Feasibility of Locating Leakages in Sewage Pressure Pipes Using the Distributed Temperature Sensing Technology. Water Air and Soil Pollution, 2017. 228(2).

[14] Arifin, B.M.S., Z. Li, S.L. Shah, G.A. Meyer, and A. Colin, A novel data-driven leak detection and localization algorithm using the Kantorovich distance. Computers \& Chemical Engineering, 2018. 108: p. 300-313.

[15] Cugueró-Escofet, M.À., V. Puig, and J. Quevedo, Optimal pressure sensor placement and assessment for leak location using a relaxed isolation index: Application to the Barcelona water network. Control Engineering Practice, 2017. 63(Supplement C): p. 1-12.

[16] Ferrandez-Gamot, L., P. Busson, J. Blesa, S. Tornil-Sin, V. Puig, E. Duviella, et al., Leak Localization in Water Distribution Networks using Pressure Residuals and Classifiers. IFAC-PapersOnLine, 2015. 48(21): p. 220-225.

[17] Kayaalp, F., A. Zengin, R. Kara, and S. Zavrak, Leakage detection and localization on water transportation pipelines: a multi-label classification approach. Neural Computing \& Applications, 2017. 28(10): p. 2905-2914.

[18] Kim, Y., S.J. Lee, T. Park, G. Lee, J.C. Suh, and J.M. Lee, Robust leak detection and its localization using interval estimation for water distribution network. Computers \& Chemical Engineering, 2016. 92(Supplement C): p. 1-17.

[19] Meseguer, J., J.M. Mirats-Tur, G. Cembrano, and V. Puig, Model-based Monitoring Techniques for Leakage Localization in Distribution Water Networks. Procedia Engineering, 2015. 119(Supplement C): p. 1399-1408.

[20] Soldevila, A., R.M. Fernandez-Canti, J. Blesa, S. Tornil-Sin, and V. Puig, Leak localization in water distribution networks using Bayesian classifiers. Journal of Process Control, 2017. 55(Supplement C): p. 1-9.

[21] Soldevila, A., J. Blesa, S. Tornil-Sin, E. Duviella, R.M. Fernandez-Canti, and V. Puig, Leak localization in water distribution networks using a mixed model-based/data-driven approach. Control Engineering Practice, 2016. 55(Supplement C): p. 162-173.

[22] Almazyad, A.S., Y.M. Seddiq, A.M. Alotaibi, A.Y. Al-Nasheri, M.S. BenSaleh, A.M. Obeid, et al., A Proposed Scalable Design and Simulation of Wireless Sensor Network-Based Long-Distance Water Pipeline Leakage Monitoring System. Sensors, 2014. 14(2): p. 3557-3577.

[23] Steffelbauer, D., M. Neumayer, M. Günther, and D. Fuchs-Hanusch, Sensor placement and leakage localization considering demand uncertainties. Procedia Engineering, 2014. 89: p. 1160-1167.

[24] Weiguo, L., W. Xiaodong, W. Haiyan, M. Changli, and W. Fenwei, A dual-sensor-based method to recognize pipeline leakage and interference signals. Journal of Loss Prevention in the Process Industries, 2015. 38: p. 79-86.

[25] Frings, J. Enhanced Pipeline Monitoring with Fiber Optic Sensors. in Proceedings of the 6th Pipeline Technology Conference, Hannover, Germany. 2011.

[26] Ren, L., T. Jiang, Z.-g. Jia, D.-s. Li, C.-1. Yuan, and H.-n. Li, Pipeline corrosion and leakage monitoring based on the distributed optical fiber sensing technology. Measurement, 2018. 122: p. 57-65.

[27] Jia, Z., L. Ren, H. Li, and W. Sun, Pipeline leak localization based on FBG hoop strain sensors combined with BP neural network. Applied Sciences (Switzerland), 2018. 8(2).

[28] Atef, A., T. Zayed, A. Hawari, M. Khader, and O. Moselhi, Multi-tier method using infrared photography and GPR to detect and locate water leaks. Automation in Construction, 2016. 61(Supplement C): p. 162-170.

[29] Revil, A., D. Mao, A.K. Haas, M. Karaoulis, and L. Frash, Passive electrical monitoring and localization of fluid leakages from wells. Journal of Hydrology, 2015. 521(Supplement C): p. 286-301.

[30] Przystałka, P. and W. Moczulski, Optimal Placement of Sensors and Actuators for Leakage Detection and Localization*. IFAC Proceedings Volumes, 2012. 45(20): p. 666-671.

[31] Sophocleous, S., D.A. Savić, Z. Kapelan, and O. Giustolisi, A Two-stage Calibration for Detection of Leakage Hotspots in a Real Water Distribution Network. Procedia Engineering, 2017. 186(Supplement C): p. 168-176.

[32] Zan, T.T.T., H.B. Lim, K.-J. Wong, A.J. Whittle, and B.-S. Lee, Event detection and localization in urban water distribution network. IEEE Sensors Journal, 2014. 14(12): p. 4134-4142.

[33] Misiunas, D., J. Vítkovský, G. Olsson, M. Lambert, and A. Simpson, Failure monitoring in water distribution networks. Water science and technology, 2006. 53(4-5): p. 503-511.

[34] Lee, S.J., G. Lee, J.C. Suh, and J.M. Lee, Online burst detection and location of water distribution systems and its practical applications. Journal of Water Resources Planning and Management, 2015. 142(1): p. 04015033.

[35] Srirangarajan, S., M. Allen, A. Preis, M. Iqbal, H.B. Lim, and A.J. Whittle, Wavelet-based burst event detection and localization in water distribution systems. Journal of Signal Processing Systems, 2013. 72(1): p. 1-16. 
[36] Liu, J., H. Su, Y. Ma, G. Wang, Y. Wang, and K. Zhang, Chaos characteristics and least squares support vector machines based online pipeline small leakages detection. Chaos, Solitons \& Fractals, 2016. 91: p. 656-669.

[37] Jensen, T.N., V. Puig, J. Romera, C.S. Kallesøe, R. Wisniewski, and J.D. Bendtsen, Leakage localization in water distribution using data-driven models and sensitivity analysis. IFAC-PapersOnLine, 2018. 51(24): p. 736-741.

[38] Steffelbauer, D.B., M. Günther, and D. Fuchs-Hanusch, Leakage Localization with Differential Evolution: A Closer Look on Distance Metrics. Procedia Engineering, 2017. 186: p. 444-451.

[39] Lu, W., W. Liang, L. Zhang, and W. Liu, A novel noise reduction method applied in negative pressure wave for pipeline leakage localization. Process Safety and Environmental Protection, 2016. 104: p. 142-149.

[40] Romero-Tapia, G., M.J. Fuente, and V. Puig, Leak Localization in Water Distribution Networks using Fisher Discriminant Analysis. IFAC-PapersOnLine, 2018. 51(24): p. 929-934. 